\title{
SOLITONS, ENVELOPE SOLITONS IN COLLISIONLESS PLASMAS
}

\author{
Y. H. ICHIKAWA
}

\author{
Institute of Plasma Physics, Nagoya University, Nagoya, Japan
}

and

\section{S. WATANABE}

\author{
Research Institute for Energy Materials, \\ Yokohama National University, Yokohama, Japan
}

\begin{abstract}
Résumé. - On présente une revue des approfondissements théoriques, numériques et expérimentaux sur la propagation d'onde non linéaire dans les plasmas sans collision. Premièrement, l'expérience historique de Ikezi et al. est discutée et comparée à une analyse théorique basée sur l'équation de Korteweg-de Vries. Un écart systématique entre l'observation et la prévision théorique suggère qu'on doit examiner des effets tels que couplage de mode d'ordre plus élevé, et contribution des particules piégées. Deuxièmement, les effets de l'amortissement de Landau non linéaire sur le soliton d'enveloppe d'onde plasma ionique sont discutés sur les bases de l'étude théorique de Ichikawa-Taniuti, de l'observation expérimentale de Watanabe et de l'analyse numérique de Yajima et al. Finalement, un type nouveau d'équation d'évolution composé pour l'onde d'Alfvéen est examiné en quelque détail. La solution rigoureuse obtenue pour ce mode représente un nouveau type de soliton d'enveloppe pour lequel à la fois la phase et l'amplitude sont sujettes à des modu lations d'extensions spatiales comparables. En conclusion, l'accent sera mis sur le fait que beaucoup plus de recherches expérimentales intensives sont attendues, puisque les méthodes puissantes pour démêler des équations d'évolution non linéaires variées sont maintenant disponibles à l'approche théorique.

Abstract. - A review is given to extensive development of theoretical, computational and experimental studies of nonlinear wave propagation in collisionless plasmas. Firstly, the historical experiment of Ikezi et al. is discussed in comparison with theoretical analysis based on the Kortewegde Vries equation. Systematic discrepancy between the observation and the theoretical prediction suggests that it is necessary to examine such as higher order mode coupling effect and contribution of trapped particles. Secondly, effects of the nonlinear Landau damping on the envelope soliton of ion plasma wave is discussed on the basis of theoretical study of Ichikawa-Taniuti, experimental observation of Watanabe and numerical analysis of Yajima et al. Finally, a new type of evolution equation derived for the Alfvén wave is examined in some detail. The rigorous solution obtained for this mode represents a new kind of envelope soliton, in which both of its phase and amplitude are subject to modulation of comparable spatial extension. In conclusion, the emphasis will be placed on the fact that much more intensive experimental researches are expected to be done, since the powerful methods to disentangle various nonlinear evolution equations are now available for theoretical approach.
\end{abstract}

1. Introduction. - Studies of nonlinear wave phenomena in collisionless plasmas provide a firm base not only for exploring fundamental researches on nonlinear physics, but also for developing practical applications in controlled nuclear fusion technology. Problems of laser-plasma interaction, anomalous transport and radio-frequency confinement are typical examples having strong motivation in the latter connection. In this paper, however, we will put our emphasis on the fact that recent advancement in understanding of nonlinear wave phenomena opens the way to establish physics of nonlinear phenomena in nature.

In the month of August 1843, Scott-Russel [1] had the first chance to observe a rounded, smooth and well defined heap of water continued its course along the channel appearently without change of form or diminution of speed. This solitary wave propagated about one miles at a rate of some eight or nine miles an hour, preserving its original figure some thirty feet long and a foot to a foot and a half in height. In 1895, analyzing competing process of dispersive effect and nonlinear steepning effect in the shallow water wave propagation, Korteweg and de Vries [2] have derived a nonlinear partial differential equation to explain the properties of the solitary wave. This equation is now called by their names.

Since Gardner and Morikawa [3] have rediscovered that the Korteweg-de Vries equation valids also for nonlinear magneto-hydrodynamic wave propagating 
perpendicular to the external magnetic field, refreshed interests have been stirred up on the studies of nonlinear wave phenomena in the field of plasma physics. Theoretical prediction of Washimi and Taniuti [4] on the possibility of the ion-acoustic solitary wave has been confirmed experimentally by Ikezi, Taylor and Baker [5], [6]. Reinforcement of the genius invention of the inverse scattering method of solving nonlinear evolution equations [7], [8] has encouraged us to endeavor to disentangle complicated nonlinear wave phenomena on the firm theoretical ground.

We begin our discussion on the historical experiment of Ikezi et al. [5], [6] on the ion-acoustic soliton in section 2 , and then proceed to discuss recent theoretical development on the properties of solitons associated with the weakly dispersive system in section 3 . In section 4, we discuss theoretical and experimental aspects of the nonlinear wave modulation in the strongly dispersive region. We present in section 5 a new type of evolution equation derived for the Alfven wave, propagating along the magnetic field, and discuss its analytic steady state solution in some details. As concluding remarks, we mention briefly potential importance of the studies of nonlinear wave phenomena on understanding of behaviour of plasmas which are expected to be produced in controlled thermonuclear fusion devices.

2. Ion-acoustic solitons. - Firstly, let us derive the Korteweg-de Vries equation for the ion-acoustic wave on the basis of the reductive perturbation theory developed by Taniuti and his collaborators during the years of $1968 \sim 1974$ [9]. For a collisionless plasma composed by cold ions and warm electrons, the basic set of equations may be expressed as (in a dimensionless form),

$$
\begin{gathered}
\frac{\partial}{\partial t} n+\frac{\partial}{\partial x}(n u)=0, \\
\frac{\partial}{\partial t} u+u \frac{\partial}{\partial x} u=-\frac{\partial}{\partial x} \psi, \\
\frac{\partial^{2}}{\partial x^{2}} \psi=n_{\mathrm{c}}-n, \\
n_{\mathrm{e}}=\exp \psi
\end{gathered}
$$

where

$$
n=\tilde{n}_{\mathrm{i}} / n_{0}, \quad n_{\mathrm{c}}=\tilde{n}_{\mathrm{c}} / n_{0}, \quad u=\tilde{u}\left(\kappa T_{\mathrm{e}} / M\right)^{-1 / 2}
$$

and

$$
\psi=\widetilde{\psi}\left(\kappa T_{\mathrm{c}} / e\right)^{-1}
$$

are the dimensionless ion number density, electron number density, ion velocity and electro-static potential, respectively. Dimensionless space-time variable $(x, t)$ are measured by the Debye distance

$$
\left(\kappa T_{\mathrm{e}} / 4 \pi e^{2} n_{\mathrm{o}}\right)^{1 / 2}
$$

and the ion plasma frequency $\left(4 \pi e^{2} n_{0} / M\right)^{1 / 2}$. Needless to say, we are considering one dimensional wave motion in the system.

Imposing the boundary condition,

$$
n=1 \quad \psi=0, \quad u=1 \quad \text { as } \quad|x| \rightarrow \infty,
$$

we introduce the stretched variables

$$
\begin{aligned}
& \xi=\varepsilon^{1 / 2}(x-t), \\
& \tau=\varepsilon^{3 / 2} .
\end{aligned}
$$

With these re-scaling of the independent variables, the basic eq. $(1 a) \sim(1 d)$ are transformed as follows,

$$
\begin{gathered}
\varepsilon \frac{\partial}{\partial \tau} n-\frac{\partial}{\partial \xi} n+\frac{\partial}{\partial \xi}(n u)=0 \\
\varepsilon \frac{\partial}{\partial \tau} u-\frac{\partial}{\partial \xi} n+u \frac{\partial}{\partial \dot{\xi}} u=-\frac{\partial}{\partial \xi} \psi \\
\varepsilon \frac{\partial^{2}}{\partial \bar{\zeta}^{2}} \psi=\exp \psi-n
\end{gathered}
$$

Substituting power series expansions of $n, u$ and $\psi$,

$$
\begin{aligned}
n & =1+\varepsilon n^{(1)}+\varepsilon^{2} n^{(2)}+\ldots \\
u & =\varepsilon u^{(1)}+\varepsilon^{2} u^{(2)}+\ldots \\
\psi & =\varepsilon \psi^{(1)}+\varepsilon^{2} \psi^{(2)}+\ldots
\end{aligned}
$$

inot eqs $(4 a) \sim(4 c)$, we can establish relationship among the first order quantities as

$$
\psi^{(1)}=n^{(1)}=u^{(1)}=n_{\mathrm{e}}^{(1)}
$$

in the lowest order expansion of eqs $(4 a) \sim(4 b)$. Their explicit $(\xi, \tau)$-dependence is determined through the Korteweg-de-Vries equation

$$
\frac{\partial}{\partial \tau} \psi^{(1)}+\frac{1}{2} \frac{\partial^{3}}{\partial \xi^{3}} \psi^{(1)}+\psi^{(1)} \frac{\partial}{\partial \xi} \psi^{(1)}=0,
$$

which is derived as the compatibility condition of the second order components of eqs $(4 a) \sim(4 c)$.

Although the Korteweg-de Vries equation can be solved analytically for an arbitrary initial value with the help of the inverse scattering method, here we present a steady state one-soliton solution of (7) as

$$
\psi=A \operatorname{sech}^{2}[D(\xi-\lambda \tau)]
$$

with

$$
\begin{aligned}
& \lambda=\frac{1}{3} A, \\
& D=(\lambda / 2)^{1 / 2} .
\end{aligned}
$$

Namely, the one soliton runs with velocity faster than the ion acoustic speed by the amount proportional to one third of its amplitude. The width of soliton is inversely proportional to the square root of its amplitude. Figure 1 illustrates nonlinear evolution of the large amplitude perturbation excited in 


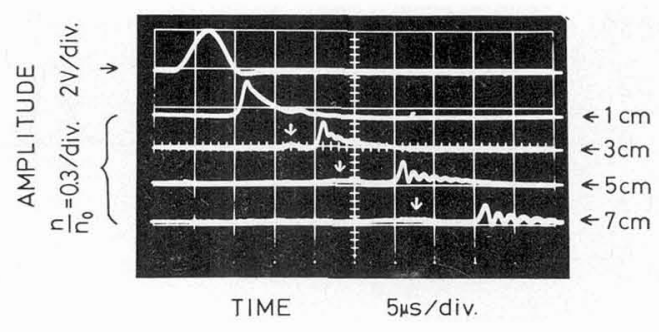

FIG. 1. - Propagation of nonlincar ion-acoustic wave. The top trace is an applied pulse. The lower traces represent subsequent decomposition of the induced perturbation into a precursor (indicated by arrows) and solitons at the distances indicated on the right.

the double plasma device, having the following parameters $n_{0}=(1 \sim 2) \times 10^{9} \mathrm{~cm}^{-3}, T_{\mathrm{e}}=2 \sim 3 \mathrm{eV}$, $T_{\mathrm{e}} / T_{\mathrm{i}} \sim 10$ in low pressure Argon gas with $(2 \sim 5) \times 10^{-4}$ torr in typical operation conditions. The large amplitude perturbation is decomposed into several peaks in the course of its propagation. The first small peak is a precursor consisted with ions reflected back from the large potential barrier, of which properties have been examined theoretically by Kato et al. [10].

We may summarize the experimental results as follows :

1) The velocity of the soliton is approximately in accord with the theoretical value predicted by $(9 a)$ but the observed velocity is faster than the velocity of the Korteweg-de Vries soliton, [5], [6], [11].

2) The width of the soliton is in rough agreement with the theoretical value of $(9 b)$, but it is narrower than the width of the Korteweg-de Vries soliton, [5], [6], [11].

3) The number of solitons is in agreement with the value predicted by the analytic solution given by the inverse scattering method, [11].

4) The recurrence to its initial form of perturbation has been demonstrated [6], [12].

Systematic discrepancy between the experimental observation and the theoretical prediction calls for refinement of simplified Korteweg-de Vries soliton description. As an improvement of the model, effects of finite ion temperature have been examined by Kato et al. [10], Tappert [13] and Tagare [14]. With regards the large amplitude effects, Schamel [15] has proposed a different type of the nonlinear equation with full account of the trapped particles by electrostatic potential of the wave, while Konno and Ichikawa [16] have shown that contribution of threewave interaction, with account of the finite ion temperature effect, removes the discrepancy between the theory and experiment considerably (Fig. 2). We should, however, emphasize that none of these can discriminate the others, conclusively. Experimental investigation for various electron-ion temperature ratio will be useful to draw definite conclusions.

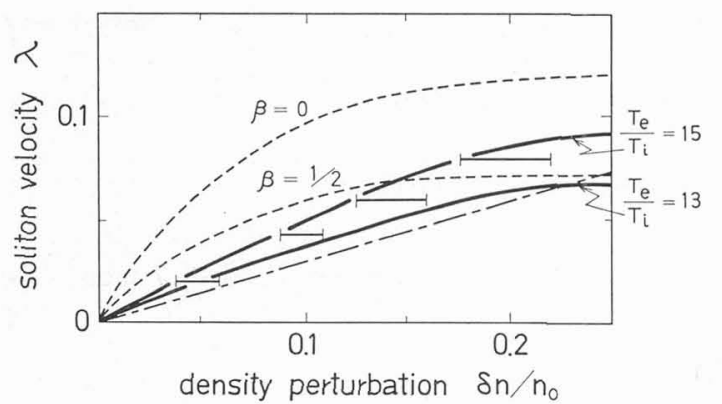

Fig. 2. - The soliton velocity as a function of amplitude of the density perturbation $\delta n / n_{0}$. The bars are experimental results taken from the reference [5]. The broken line with dots is for the Korteweg-de-Vries soliton. The dotted lines are curves. Calculated for the reference [15] with arbitrary parameter of $\beta=T_{\mathrm{e}} / T_{\mathrm{p}}$, where $T_{\mathrm{t}}$ is temperature of trapped electrons. The heavy curves are results of the reference [16].

3. Higher order perturbation and dressed soliton. Besides the above mentioned refinements from the physical consideration, we may ask how contributions of higher order perturbation terms modify basic properties of the Korteweg-de Vries soliton within the mathematical framework of the model system described by eqs. $(1 a) \sim(1 d)$. We have undertaken the analysis of higher order terms of eqs. $(4 a) \sim(4 c)$ [17]. The second order quantities $n^{(2)}$ and $u^{(2)}$ are expressed as

$$
\begin{aligned}
& n^{(2)}=\psi^{(2)}+\frac{1}{2} \psi^{(1)} \psi^{(1)}-\frac{\partial^{2}}{\partial \xi^{2}} \psi^{(1)}, \\
& u^{(2)}=\psi^{(2)}-\frac{1}{2} \cdot \frac{\partial^{2}}{\partial \xi^{2}} \psi^{(1)},
\end{aligned}
$$

while behaviour of the second order potential $\psi^{(2)}$ is determined from the following equation,

$$
\frac{\partial}{\partial \tau} \psi^{(2)}+\frac{1}{2} \frac{\partial^{3}}{\partial \xi^{3}} \psi^{(2)}+\frac{\partial}{\partial \xi}\left(\psi^{(1)} \psi^{(2)}\right)=S\left(\psi^{(1)}\right)
$$

where

$$
\begin{aligned}
S\left(\psi^{(1)}\right)=-\frac{3}{8} \frac{\partial^{5}}{\partial \xi^{5}} \psi^{(1)}+ & \frac{1}{2} \psi^{(1)} \frac{\partial^{3}}{\partial \xi^{3}} \psi^{(1)}- \\
& -\frac{5}{8} \frac{\partial}{\partial \xi}\left(\frac{\partial}{\partial \xi} \psi^{(1)}\right)^{2}
\end{aligned}
$$

Thus, the Korteweg-de Vries eq. (7) and the linear inhomogeneous eq. (11a) with $(11 b)$ describe nonlinear ion acoustic wave propagation in the second order.

Seeking a type of solutions $\psi^{(1)}(\eta)$ and $\psi^{(2)}(\eta)$ with argument

$$
\eta=\xi-\lambda \tau,
$$

we have obtained a steady one soliton solution of the coupled set of eqs. (7) and (11a) with $(11 b)$ as follows, letting ordering parameter $\varepsilon \rightarrow 1$, 


$$
\begin{aligned}
\psi(\eta)= & \psi^{(1)}(\eta)+\psi^{(2)}(\eta), \\
\psi^{(1)}(\eta)= & 3 \lambda \operatorname{sech}^{2}(D \eta), \\
\psi^{(2)}(\eta)= & \frac{9}{4} \lambda^{2} \operatorname{sech}^{2}(D \eta) \times \\
& \times\left\{2 D \eta \tanh (D \eta)-8+7 \operatorname{sech}^{2}(D \eta)\right\},
\end{aligned}
$$

with

$$
D=(\lambda / 2)^{1 / 2} \text {. }
$$

The perturbed potential $\psi(\eta)$ can be regarded as the dressed soliton, of which velocity $\lambda$ is given by the amplitude $A$ of the ion acoustic potential perturbation as

$$
\lambda=\frac{1}{3} A+\frac{1}{12} A^{2}
$$

We have observed numerically that the steady state clouds (13c) moves stably with the Korteweg-de Vries soliton core $(13 b)$.

We have also examined numerically the collision processes of the dressed solitons [18]. As a solution of the Korteweg-de Vries eq. (7), we take the wellknown two-soliton solution,

$$
\begin{aligned}
\psi^{(1)}(\xi, \tau)=24 & {\left[1+\exp \left(2 D_{1} \eta_{1}\right)+\exp \left(2 D_{2} \eta_{2}\right)+\right.} \\
& \left.+\exp \left(2 D_{1} \eta_{1}+2 D_{2} \eta_{2}+\delta_{3}\right)\right]^{-1} \\
& \times\left[D_{1}^{2} \exp \left(2 D_{1} \eta_{1}\right)+D_{2}^{2} \exp \left(2 D_{2} \eta_{2}\right)\right. \\
& +2\left(D_{1}-D_{2}\right)^{2} \exp \left(2 D_{1} \eta_{1}+2 D_{2} \eta_{2}\right) \\
& +D_{2}^{2} \exp \left(4 D_{1} \eta_{1}+2 D_{2} \eta_{2}+\delta_{3}\right)
\end{aligned}
$$

$$
\left.+D_{1}^{2} \exp \left(2 D_{1} \eta_{1}+4 D_{2} \eta_{2}+\delta_{3}\right)\right] \text {, }
$$

where

$$
\begin{aligned}
& \eta_{1}=\xi-\lambda_{1} \tau-\delta_{1}, \\
& \eta_{2}=\xi-\lambda_{2} \tau-\delta_{2}, \\
& D_{1}=\left(\lambda_{1} / 2\right)^{1 / 2}, \\
& D_{2}=\left(\lambda_{2} / 2\right)^{1 / 2}, \\
& \delta_{3}=\log \left[\frac{\left(D_{1}-D_{2}\right)^{2}}{\left(D_{1}+D_{2}\right)^{2}}\right] .
\end{aligned}
$$

The parameters $\delta_{1}$ and $\delta_{2}$ denote initial positions of the two solitons. We describe an initial state of the binary system of the dressed solitons approximately by superposing the steady state second order clouds (13c) with the amplitude given by $\lambda_{1}$ and $\hat{\lambda}_{2}$ at the positions of $\delta_{1}$ and $\delta_{2}$, respectively. Figure 3 presents the temporal evolution of the shape of two dressed solitons with the values of $\delta_{1}=0.3$ and $\delta_{2}=0.24$. The thin line represents the Korteweg-de Vries soliton core, while the broken line at time $\tau=0$ represents the steady state second order clouds asso-

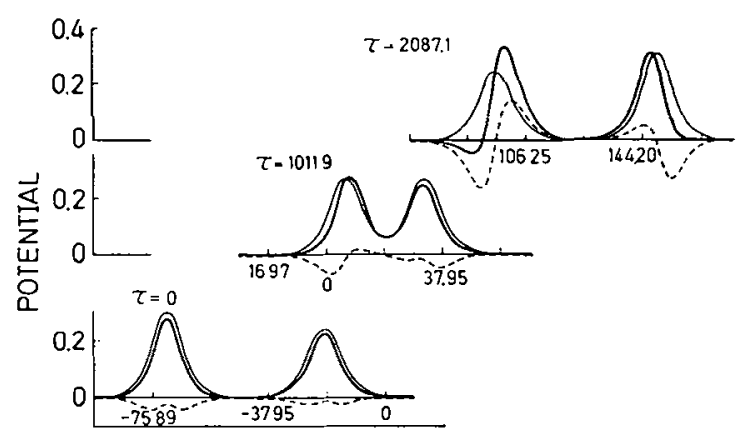

Fic. 3. - Collision process of two dressed solitons with $\lambda_{1}=0.3$ and $\lambda_{2}=0.24$, represented by the heavy lines. The thin lines indicate the Korteweg-de Vries soliton cores, while the dotted lines represent the second order clouds.

ciated with each soliton core. In the course of collision process, we observe that the clouds associated with the binary soliton core redistribute themselves in such a way to equalize their amplitude after the collision.

Concerning with the structure of the dressed soliton, Sugimoto and Kakutani [19] have remarked that the term with $D \eta \tanh (D \eta)$ implies the fact that the reductive perturbation expansion carried up to the second order is not free from the secularity. They have proposed to eliminate this term by the method of multiple space-time variables. Introducing the following multiple space-time variables.

$$
\begin{array}{lll}
\xi=\varepsilon^{1 / 2}(x-t), & \tau=\varepsilon^{3 / 2} t, \\
\xi_{2}=\varepsilon^{3 / 2}(x-t), & \tau_{2}=\varepsilon^{5 / 2} t,
\end{array}
$$

they have obtained the following equation for the place of $(11 a)$,

$$
\begin{aligned}
\frac{\partial}{\partial \tau} \psi^{(2)} & +\frac{1}{2} \frac{\partial^{3}}{\partial \xi^{3}} \psi^{(2)}+\frac{\partial}{\partial \xi}\left(\psi^{(1)} \psi^{(2)}\right)=S\left(\psi^{(1)}\right)- \\
- & {\left[\frac{\partial}{\partial \tau_{2}} \psi^{(1)}-\frac{1}{2} \frac{\partial}{\partial \xi_{2}}\left(\psi^{(1)}\right)^{2}-\frac{3}{2} \frac{\partial^{3}}{\partial \xi_{2} \partial \xi^{2}} \psi^{(1)}\right], }
\end{aligned}
$$

where $S\left(\psi^{(1)}\right)$ is given by $(11 b)$. As for the steady one soliton solution of eqs. (11a) and (18), taking a form of

$$
\begin{array}{r}
\psi^{(1)}\left(\eta ; \tau_{2}, \xi_{2}\right)=6 D\left(\tau_{2}, \xi_{2}\right)^{2} \operatorname{sech}^{2} \\
{\left[D\left(\tau_{2}, \xi_{2}\right)\left(\eta+\theta\left(\tau_{2}, \xi_{2}\right)\right)\right],}
\end{array}
$$

where $\eta$ is given by (12), and $D$ is defined by $(13 d)$, they have obtained the following set of equations,

$$
\begin{gathered}
\cdot \frac{\partial}{\partial \tau_{2}} D=0, \quad \frac{\partial}{\partial \xi_{2}} D=0 \\
\frac{\partial}{\partial \tau_{2}} \theta=3 D^{4}, \quad \frac{\partial}{\partial \xi_{2}} \theta=-\frac{3}{2} D^{2},
\end{gathered}
$$

as conditions to eliminate the secular term. Hence, slow variation of the phase $\theta\left(\tau_{2}, \xi_{2}\right)$ is given by 


$$
\theta\left(\tau_{2}, \xi_{2}\right)=-\frac{3}{2} D^{2}\left(\xi_{2}-\lambda \tau_{2}\right)=-\varepsilon \frac{3}{2} D^{2} \eta,
$$

were $D=(\lambda / 2)^{1 / 2}$ is a constant. Solving $\psi^{(2)}$ from (18) with (19) and (21), one can easily write down the perturbed potential up to the second order terms as

$$
\begin{aligned}
\widetilde{\psi}(\eta)=3 \lambda \operatorname{sech}^{2}(\tilde{D} \eta) & +\frac{9}{4} \lambda^{2} \operatorname{sech}^{2}(\widetilde{D} \eta) \times \\
\times & {\left[-8+7 \operatorname{sech}^{2}(\tilde{D} \eta)\right], }
\end{aligned}
$$

with the definition of

$$
\widetilde{D}=(\lambda / 2)^{1 / 2}\left(1-\frac{3}{4} \lambda\right) .
$$

It should be noticed here that the velocity of soliton $\lambda$ is given by (14) expressed in terms of the maximum soliton amplitude $A$ as before, but structure of the renormalized soliton is now given as

$$
\begin{aligned}
\widetilde{\psi}(\eta) & =\widetilde{\psi}_{\mathrm{co}}(\eta)+\tilde{\psi}_{\mathrm{cl} 1}(\eta), \\
\left.\tilde{\psi}_{\mathrm{co}} \eta\right) & =3 \lambda\left(1-\frac{3}{4} \lambda\right) \operatorname{sech}^{2}(\widetilde{D} \eta), \\
\tilde{\psi}_{\mathrm{cl}}(\eta) & =-7 \cdot \frac{9}{4} \lambda^{2} \operatorname{sech}^{2}(\tilde{D} \eta) \tanh ^{2}(\widetilde{D} \eta),
\end{aligned}
$$

where $\widetilde{\psi}_{\text {co }}(\eta)$ is the second orther renormalized soliton core, and $\widetilde{\psi}_{\mathrm{cl}}(\eta)$ represents the second order cloud surrounding the core. In figure 4 , we illustrate the structure of the renormalized soliton.

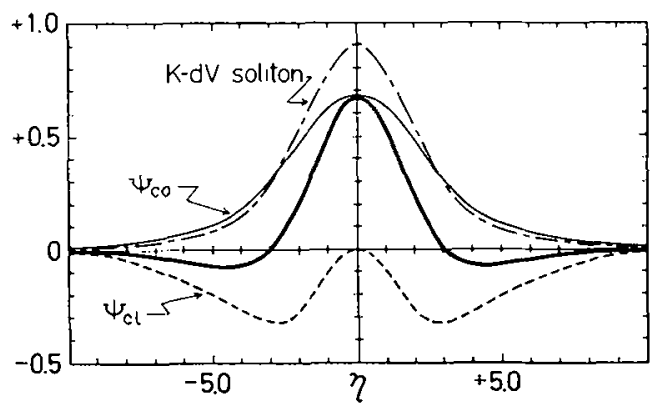

FIG. 4. - Structure of renormalized dressed soliton. The heavy line represents shape of eq. (24a) for $\lambda=0.3$, while the thin line is eq. (24b) and the dotted line eq. (24c), respectively.

Now, Kodama and Taniuti [20] have developed an elegant analysis of the renormalization procedure in carrying out the reductive perturbation ot arbitrary higher order terms. They have reduced a set of equations for a model system to a renormalized Kortewegde Vries equation, and have shown explicitly that the renormalization can be carried out not only for the one soliton state, but also for the system with an arbitrary number of solitons. Thus, we are now standing at a position where we can investigate dynamical properties of the renormalized soliton systems on firm ground.
4. Self-modulation of strongly dispersive waves. We now turn our interests to phenomena of selfmodulation of a quasi-monochromatic wave in strongly dispersive region such as the electron Langmuir wave, the ion plasma wave and the whistler wave in mangetized plasmas. The problem has close connection with such phenomena of self-focusing and self-contraction of wave packets in nonlinear optics, and modulation-instability of the gravity waves on water. In collisionless plasmas, it is well awared by us that the resonant wave-particle interaction at the phase velocity causes very different nonlinear modulation associated with the trapped particles. Nevertheless, we develope our disscussion for a special case in which the trapped particles do not give rise to appreciable effects.

Taniuti et al. [21] have presented a systematic analysis of the nonlinear modulation of a quasimonochromatic wave by examining a system of equations

$$
\frac{\partial}{\partial t} U+A(U) \frac{\partial}{\partial x} U+B(U)=0,
$$

where $U$ is a column vector with $n$-components $u_{1}$, $u_{2}, \ldots, u_{n}$ and $A$ an $n \times n$ matrix and $B$ a column vector. The set of eqs. $(1 a) \sim(1 d)$ can be reduced to the standard form by setting $-\delta \psi / \delta x=E$. It is assumed that $(25)$ has a constant state solution $U_{0}$, which satisfies

$$
B\left(U_{0}\right)=0 .
$$

Considering a plane wave of infinitesimal amplitude propagating in the constant state $U_{0}$, we assume that $U$ can be expanded about $U_{0}$ as

$U=U_{0}+\sum_{i=-\infty}^{\infty} \sum_{n=1}^{\infty} \varepsilon^{n} U_{l}^{(n)}(\xi, \tau) \exp [i l(k x-\omega t)]$,

where $\varepsilon$ measures the size of perturbed amplitude and $(\xi, \tau)$ are the stretched space-time variables defined as

$$
\begin{aligned}
& \xi=\varepsilon(x-\lambda t), \\
& \tau=\varepsilon^{2} t .
\end{aligned}
$$

Substitution of eqs. (28a) $\sim(28 b)$ with $(27)$ into the original eq. (25) yields a set of equations corresponding to the each oder of powers of $\varepsilon$ and the $l$-th harmonie component. In the first order of $\varepsilon$, the linear dispersion relation

$$
\operatorname{det}\left[ \pm i\left(\omega I-k \mathrm{~A}\left(U_{0}\right)\right)+\nabla_{u} B(U)_{u=u_{0}}\right]=0,
$$

assures that $U_{ \pm \text {I }}^{(1)}$ can be expressed as

$$
U_{ \pm 1}^{(1)}(\xi, \tau)=\psi(\xi, \tau) R
$$

with the right eigenvector $R$ given by

$$
\left[\mp i\left(\omega I-k A\left(u_{0}\right)\right]+\left[\nabla_{u} B(u)_{u=u_{0}}\right] R=0,\right.
$$


and $U_{i}^{(1)}=0$ for $|l| \neq 1$. In the second order of $\varepsilon$, the $l=1$ component yields a condition

$$
\hat{\lambda} \equiv \frac{\partial \omega(k)}{\partial k}
$$

to deal with nontrivial case $\partial U_{ \pm 1}^{(1)} / \partial \xi \neq 0$. The $l=2$ and $l=0$ component of the second order equation determines the second order beat wave $U_{2}^{(2)}$ and $U_{0}^{(2)}$, respectively. Finally, at the third order of $\varepsilon$, the $l=1$ component gives rise to the nonlinear Schrödinger equation,

$$
i \frac{\partial}{\partial \tau} \psi+p \frac{\partial^{2}}{\partial \xi^{2}} \psi+q|\psi|^{2} \psi=0
$$

where $p=(1 / 2) \hat{o}^{2} \omega / \partial k^{2}$ represents the dispersion effect, while $q$ measures the strength of nonlinearity. For the ion plasma wave propagating in a system described by eqs. $(1 a) \sim(1 d)$, we get [22]

$$
\begin{aligned}
p=-\frac{3}{2} \omega\left(\frac{\omega}{k}\right)^{4} & \\
q=\frac{\omega}{2}\left[-\frac{1}{k^{2}}\right. & -\frac{k^{2}}{2 \omega^{2}}+\frac{\omega^{4}}{2 k^{4}}+\frac{\omega^{4}}{3 k^{6}}-\frac{\omega^{6}}{3 k^{6}}+ \\
& \left.+\frac{1}{1-(\omega / k)^{3}} \frac{\omega^{2}}{k^{2}}\left(1+\frac{\omega^{2}}{k^{2}}\right)^{2}\right] .
\end{aligned}
$$

Since $q$ given by $(34 b)$ is positive, the coefficients $p$ and $q$ take the opposite sign.

When $p q<0$, finite amplitude plane wave is stable against modulation. For this case, setting

$$
\psi=\sqrt{\rho(\xi, \tau)} \exp \left[\frac{i}{2 p} \int^{\xi} \sigma\left(\xi^{\prime}, \tau\right) \mathrm{d} \xi^{\prime}\right]
$$

we obtain the following soliton solution,

$$
\rho(\xi, \tau)=\rho_{0}\left[1-A \operatorname{sech}^{2}\left\{\frac{C}{2 p} A^{1 / 2}\left(\xi-\lambda_{ \pm} \tau\right)\right\}\right]
$$

$$
\begin{aligned}
& \sigma(\xi, \tau)=\lambda_{ \pm} \mp C(1-A)^{1 / 2} \times \\
& \times\left[1-A \operatorname{sech}^{2}\left\{\frac{C}{2 p} A^{1 / 2}\left(\xi-\lambda_{ \pm} \tau\right)\right\}\right]^{-1}
\end{aligned}
$$

with

$$
\begin{aligned}
& \lambda_{ \pm}=\sigma_{0} \pm C(1-A)^{1 / 2} \\
& C=\left(-2 p q \rho_{0}\right)^{1 / 2}
\end{aligned}
$$

This type of envelope soliton is called as a dark soliton reffering to the nonlinear optics.

On the other hand, when the coefficients $p$ and $q$ take the same sign, $i$. $e ., p q>0$, the wave is modulationally unstable in the sense that the finite amplitude plane wave breaks up to a train of solitons. For this case, eq. (33) has an envelope-soliton solution, which satisfies the boundary condition that $\psi(\xi, \tau)$ and its derivatives vanish at $\xi= \pm \infty$,

$$
\begin{aligned}
\psi(\xi, \tau)=A \operatorname{sech} & {\left[\left(\frac{q}{2 p}\right)^{1 / 2} A(\xi-V \tau)\right] \times } \\
\times & \exp \left[i\left(\frac{V}{2 p} \xi-\frac{V^{2}}{4 p} \tau+\frac{1}{2} q A^{2} \tau\right)\right],
\end{aligned}
$$

where an arbitrary constant $V$ defines the velocity of the envelope soliton.

Now, it has been emphasized by Ikezi and Kiwamoto [23] that nonlinear Landau damping processes play important part in the phenomena of nonlinear propagation of the ion plasma wave. Therefore, we have examined carefully contribution of the resonance particles at the group velocity by formulating the problem on basis of the Vlasov description of collisionless plasmas [24]. It has been found that the wavewave-particle resonant interaction modifies drastically contribution of the slow beat wave, i. e., the second order $l=0$ component in the expansion scheme of eq. (27), and gives rise to the modified nonlinear Schrödinger equation with a nonlocal-nonlinear integral term,

$$
\begin{aligned}
i \frac{\partial}{\partial \tau} \psi+p \frac{\partial^{2}}{\partial \xi^{2}} \psi & +q|\psi|^{2} \psi+ \\
& +r \frac{P}{\pi} \int \frac{\left|\psi\left(\xi^{\prime}, \tau\right)\right|^{2}}{\xi-\xi^{\prime}} \mathrm{d} \xi^{\prime} \psi=0 .
\end{aligned}
$$

The linear stability of (38) can be examined by linearization of (35) given as

$$
\begin{gathered}
\rho=\rho_{0}+\{\delta \rho \exp [i(K \xi-\Omega \tau)]+ \\
\quad+\text { complex conjugate }\}, \\
\sigma=\quad\{\delta \sigma \exp [i(K \delta-\Omega \tau)]+ \\
+ \text { complex conjugate }\} .
\end{gathered}
$$

The dispersion relation reduced from (38) determines

$$
\Omega=\Omega_{\mathrm{r}}(K)+i \Gamma(K)
$$

where

$$
\begin{array}{r}
\Omega_{\mathrm{r}}= \pm \frac{1}{\sqrt{2}}\left[\left\{\left(p^{2} K^{2}-2 p q \rho_{0}\right)^{2}+\left(2 p r \rho_{0}\right)^{2}\right\}^{1 / 2}+\right. \\
\left.\quad+\left(p^{2} K^{2}-2 p q \rho_{0}\right)\right]^{1 / 2}|K|, \quad(40 b) \\
\Gamma=\mp \frac{1}{\sqrt{2}}\left[\left\{\left(p^{2} K^{2}-2 p q \rho_{0}\right)^{2}+\left(2 p r \rho_{0}\right)^{2}\right\}^{1 / 2}-\right. \\
\left.-\left(p^{2} K^{2}-2 p q \rho_{0}\right)\right]^{1 / 2}|K|
\end{array}
$$

In the small amplitude limit $|p / 2 q| K^{2} \gg \rho_{0}$, this is reduced correctly to the nonlinear Landau damping process, in which the wave energy is transferred from the higher frequency side band to the lower frequency side band. 
When $p q>0$, eq. (40c) takes maximum growth rate

$$
\Gamma_{\mathrm{m}}=\mp\left(q^{2}+r^{2}\right)^{1 / 2} \rho_{0}
$$

with the maximum frequency shift

$$
\Omega_{\mathrm{m}}= \pm \frac{r}{q}\left(q^{2}+r^{2}\right)^{1 / 2} \rho_{0}
$$

for the value of wave number

$$
K_{\mathrm{m}}=\left\{\left(q^{2}+r^{2}\right) / p q\right\}^{1 / 2} \sqrt{\rho_{0}} .
$$

On the other hand, if $p q<0$, in the large amplitude limit of $\rho_{0} \gg|p / 2 q| K^{2}$, eqs. (40b) and (40c) take the asymptotic values

$$
\begin{aligned}
& \Omega_{\mathrm{r}}= \pm\left\{\left(q^{2}+r^{2}\right)^{1 / 2}+|q|\right\}^{1 / 2} \sqrt{|p| K^{2} \rho_{0}} \\
& \Gamma=\mp\left\{\left(q^{2}+r^{2}\right)^{1 / 2}-|q|\right\}^{1 / 2} \sqrt{|p| K^{2} \rho_{0}} .
\end{aligned}
$$

Above analysis illustrates that the contribution of wave-wave-particle resonance at the group velocity leads to modulational instability regardless the sign of $p q$.

Experimental investigations of the modulational instability war carried out for the ion acoustic wave [25]. Figure 5 shows a transition from the linear to nonlinear propagation of ion acoustic wave packets in the typical low pressurs Argon plasma $(1 \sim 2) \times 10^{-4}$ torr, with $n_{\mathrm{e}}=(1 \sim 2) \times 10^{9} \mathrm{~cm}^{-3}$, $T_{\mathrm{e}}=(1.5 \sim 2.0) \mathrm{eV}$ and $T_{\mathrm{e}} / T_{\mathrm{i}}=10 \sim 12$. The initial

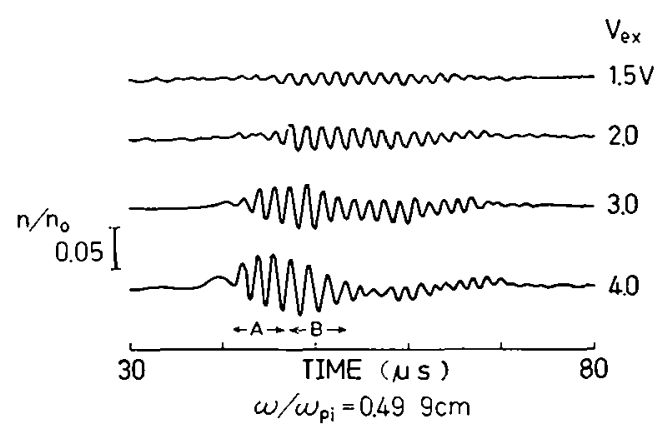

Fig. 5. - Nonlinear modulation of ion-acoustic wave packet observed in the reference [25].

profile of an envelope has $30 \mu$ s duration, in which the amplitude increases linearly in the first $10 \mu \mathrm{s}$, then is kept constant for the subsequent $10 \mu$ s and falls down linearly to zero in the last $10 \mu \mathrm{s}$. The carrier frequency is about $0.5 \omega_{\mathrm{pj}}$. Wave packets are excited in a plasma by a conventional grid exciter and are recieved by a plane probe at $9 \mathrm{~cm}$ from the grid. For a small amplitude of exciting voltage, $V_{\mathrm{cx}}=1.5 \mathrm{~V}$, the recieved wave form resembles the input one, except that the frequency in the envelope tail is slightly higher than that in the front, indicating manifestation of the dispersion effect. The profile of the envelope changes drastically when the amplitude of wave packets increases. That is, at $V_{\text {ex }}=2.0 \mathrm{~V}$ the wave front steepens, and then the modulational instability sets in as can be seen from the wave patterns at $V_{c x}=3.0 \mathrm{~V}$ and $V_{c x}=4.0 \mathrm{~V}$. In the bottom trace, the initial wave packet is divided into three parts and the amplitude of the first region is largely enhanced.

Let us make an important remark on the largest wave packet in the bottom trace. We recognize that the frequency in the region $\mathrm{A}$ where the amplitude builds up with time is higher than the frequency in the region $\mathbf{B}$ where the amplitude diminishes with time. This shift in frequency indicates that the large amplitude wave propagates more rapidly than the small amplitude wave, providing an evidence for the nonlinear dispersion effect. The group velocity dispersion of the ion wave, on the other hand, makes the velocity of the high frequency part (region A) slower than that of the low frequency part (region B). Thus, as a result of competition between nonlinear dispersion and group velocity dispersion, the modulational instability takes place in the ion wave propagation. This is the reason why we have observed the modulational instability of the ion wave. In the bottom trace, the frequency shift is found to be $\left|\Delta \omega / \omega_{0}\right|=0.15$, which is about two times larger than the shift calculated from eq. (40). This discrepancy is plausible, because, in the experiment, the envelope amplitude damps spatially and there exists ambiguity in determining the amplitude experimentally.

Recently, Ikezi et al. [26] have examined the modutation of ion waves, and concluded that the modulational instability does not take place, but effects of trapped particles are essential. In order to clearify the discrepancy between their observation and our results, it is necessary to re-examine experimental condition such as effects of ion collision, presence of noise in a plasma.

Having shown an experimental evidence of the nonlinear wave modulation of the ion plasma wave, we close discussion of the present section by illustrating results of numerical analysis of eq. (38) carried out by Yajima et al. [27]. Restricting our interest to the case of $p q>0$, we examine how the envelope soliton given as (37) deforms under the action of the nonlocal-nonlinear integral term of eq. (38). Figure 6 shows the numerical solutions of eq. (38) with initial value

$$
\psi(\xi, \tau=0)=A \operatorname{sech}\left[\left(\frac{q}{2 p}\right)^{1 / 2} A \xi\right]
$$

where we take $A=q=2 p=1$ for simplicity. The value of $r$ is arbitrary chosen to be $r=0.5$. We can see that the soliton deforms in asymmetric way and comes to run towards the positive direction. Figure 7 


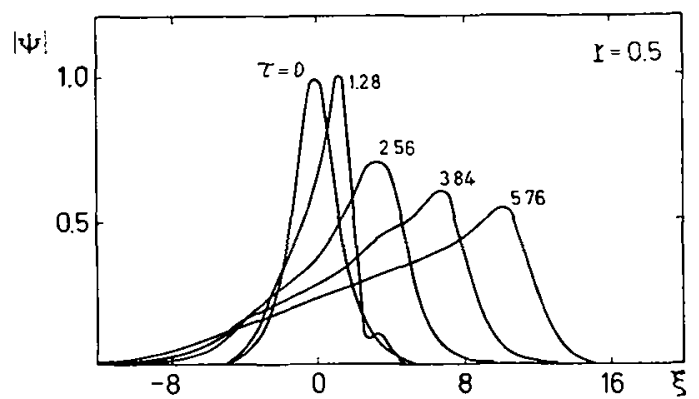

FIG. 6. - Temporal evolution of the envelope soliton under action of the wave-wave-particle resonant interaction.

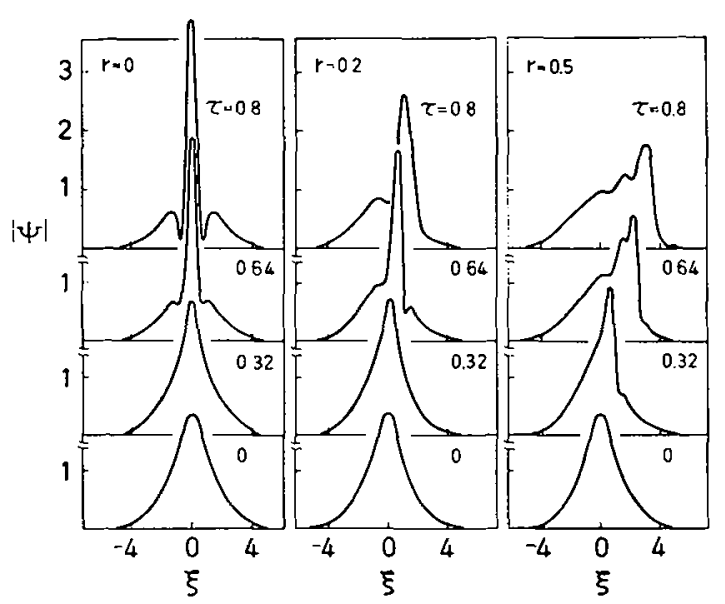

FIG. 7. - Effect of the wave-wave-particle interaction upon the bound state of thrce envelope solitons. The left figure shows evolution of envelope of eq. (44), while the other two figures represent distortion of the cnvelope due to the nonlocal-nonlinear term of eq. (38).

shows effects of nonlinear Landau damping on bound state of envelope solitons. Eq. (33) has a solution

$$
\begin{aligned}
& \psi(\xi, \tau)=4 A \exp \left[i q A^{2} \tau / 2\right] \times \\
& \times \frac{\cosh (3 B \xi)+3 \cosh (B \xi) \exp \left(4 i q A^{2} \tau\right)}{\cosh (4 B \xi)+4 \cosh (2 B \xi)+3 \cos \left(4 q A^{2} \tau\right)}
\end{aligned}
$$

which satisfies the initial condition

$$
\begin{gathered}
\psi(\xi, \tau=0)=2 A \operatorname{sech}(B \xi) \\
B=(q / 2 p)^{1 / 2} A .
\end{gathered}
$$

This solution does not decay into a train of solitons, but pulsates with a period $\pi /\left(2 q A^{2}\right)$. Numerical solutions of eq. (38) for the initial condition (44a) indicate that solitons bounded in its initial state are made to be free, and each solitons travels with changing their shape and velocity. Associated with the gain of velocity of soliton, the resonant particles at the group velocity will be ejected to the opposite direction as a bunch of particles.

5. Circular polarized nonlinear Alfvén waves. Investigation of properties of the Alfvén waves in a gaseous plasma attracts particular interests in connection with search for useful methods to heat a plasma [28]. In the problems of space physics, large amplitude incompressible magnetic field perturbation observed in the solar wind has been attributed to propagation of the Alfven wave [29], and has inspired theoretical analysis of possible existence of an exact solitary Alfvén wave [30].

In their systematic analysis of nonlinear hydromagnetic waves, Kakutani and Ono [31] have shown that, as far as the waves are propagating at an angle with a uniform external magnetic field, the nonlinear magneto-acoustic wave is described by the Kortewegde Vries equation, while propagation of the nonlinear Alfvén wave is described by the modified Korteweg-de Vries equation. However, it has been noticed firstly by Kawahara [32] that when the hydromagnetic wave is propagating along the external magnetic field these equations cease to be valid, because the dispersion relations for the magnetoacoustic wave and the Alfvén wave are degenerate in the long wave length limit for the parallel propagation. He obtained a modified type of nonlinear Schrödinger equation. Since the equation derived by Kawahara represents a new type of evolution equation, we will describe briefly derivation of this equation, and then discuss its stationary exact solutions, which represent new types of envelop solitons $\left({ }^{1}\right.$ ).

Neglecting the effects of displacement current and charge separation, we can reduce the system of equation for cold plasma to the fundamental equations for one dimensional propagation in dimensionless form,

$$
\begin{gathered}
\frac{\partial}{\partial t} n+\frac{\partial}{\partial x}(n u)=0 \\
\frac{\mathrm{d}}{\mathrm{d} t} u+n^{-1} \frac{\partial}{\partial x}\left\{\frac{1}{2}\left(B_{y}^{2}+B_{z}^{2}\right)\right\}=0
\end{gathered}
$$

$$
\begin{array}{r}
\frac{\mathrm{d}}{\mathrm{d} t} v-n^{-1} \frac{\partial}{\partial x} B_{y}=-R_{\mathrm{e}}^{-1} \frac{\mathrm{d}}{\mathrm{d} t}\left(n^{-1} \frac{\partial}{\partial x} B_{z}\right), \\
\frac{\mathrm{d}}{\mathrm{d} t} w-n^{-1} \frac{\partial}{\partial x} B_{z}=R_{\mathrm{c}}^{-1} \frac{\mathrm{d}}{\mathrm{d} t}\left(n^{-1} \frac{\partial}{\partial x} B_{y}\right), \\
\frac{\mathrm{d}}{\mathrm{d} t} B_{y}-\frac{\partial}{\partial x} v+B_{y} \frac{\partial}{\partial x} u=R_{\mathrm{i}}^{-1} \frac{\partial}{\partial x}\left(\frac{\mathrm{d}}{\mathrm{d} t} w\right),
\end{array}
$$

$\frac{\mathrm{d}}{\mathrm{d} t} B_{z}-\frac{\partial}{\partial x} w+B_{z} \frac{\partial}{\partial x} u=-R_{\mathrm{j}}^{-1} \frac{\partial}{\partial x}\left(\frac{\mathrm{d}}{\mathrm{d} t} v\right)$,

where $\mathrm{d} / \mathrm{d} t: \equiv \partial / \partial t+u . \partial / \partial x, \quad \mathbf{v}=(u, v, w)$ denotes the velocity of electrons, $n$ the density of electrons, $\mathbf{B}=\left(B_{x}=1 . B_{y}, B_{z}\right)$ the magnetic induction vector, $R_{\mathrm{e}}$ and $R_{\mathrm{f}}$ represent ratios of the electron and the ion cyclotron frequencies to the characteristic frequency, respectively. The above system has a linear dispersion relation

$$
\frac{\omega}{k}=1 \pm \mu k,
$$

(1) E. Mjølhus has called our attention to the fact that this type of nonlinear evolution equation has been derived firstly by Rogister A., Phys. Fluids 14 (1971), 2733. 
where

$$
\mu=\frac{1}{2}\left(R_{\mathrm{i}}^{-1}-R_{\mathrm{e}}^{-1}\right)
$$

The double sign \pm designates the right $(+)$ and left (-) polarized Alfvén waves, of which amplitudes are given as

$$
\begin{aligned}
& \Phi_{\mathrm{R}}=B_{y}-i B_{y}, \\
& \Phi_{\mathrm{L}}=B_{y}+i B_{z} .
\end{aligned}
$$

respectively. As for stretching of the space-time variables is concerned, in accord with the linear dispersion relation $(46 a)$, we introduce the stretched spacetime variables

$$
\begin{aligned}
\xi & =\varepsilon(x-t), \\
\tau & =\varepsilon^{2} t,
\end{aligned}
$$

assuming $k \sim 0(\varepsilon)$. We expand the variables, on the other hand, in accord with Kakutani and Ono as

$$
\begin{aligned}
n & =1+\varepsilon n^{(1)}+\varepsilon^{2} n^{(2)}+\cdots \\
u & =\varepsilon u^{(1)}+\varepsilon^{2} u^{(2)}+\cdots \\
v & =\varepsilon^{1 / 2}\left(v^{(1)}+\varepsilon v^{(2)}+\cdots\right) \\
w & =\varepsilon^{1 / 2}\left(w^{(1)}+\varepsilon w^{(2)}+\cdots\right) \\
B_{y} & =\varepsilon^{1 / 2}\left(B_{y}^{(1)}+\varepsilon B_{y}^{(2)}+\cdots\right) \\
B_{z} & =\varepsilon^{1 / 2}\left(B_{z}^{(1)}+\varepsilon B_{z}^{(2)}+\cdots\right)
\end{aligned}
$$

Substituting eqs. $(49 a) \sim(49 f)$ with the transformation of eqs. $(48 a) \sim(48 b)$, we get the following relationships among the first order quantities,

$$
\begin{aligned}
& v^{(1)}=-B_{y}^{(1)}, \quad w^{(1)}=-B_{z}^{(1)} \\
& n^{(1)}=u^{(1)}=\frac{1}{2}\left\{B_{y}^{(1)^{2}}+B_{z}^{(1)^{2}}\right\} .
\end{aligned}
$$

Eliminating the second order quantities from set of equations at the order of $\varepsilon^{5 / 2}$, we obtain simply the nonlinear evolution equation for the right polarized Alfvén wave

$$
\frac{\partial}{\partial \tau} \varphi_{\mathrm{R}}+\frac{1}{4} \frac{\partial}{\partial \xi}\left\{\left|\varphi_{\mathrm{R}}\right|^{2} \varphi_{\mathrm{R}}\right\}-i \mu-\frac{\partial^{2}}{\partial \xi^{2}} \varphi_{\mathrm{R}}=0,
$$

and for the left polarized Alfvén wave,

$$
\frac{\partial}{\partial \tau} \varphi_{L}+\frac{1}{4} \frac{\partial}{\partial \xi}\left\{\left|\varphi_{L}\right|^{2} \varphi_{L}\right\}+i \mu \frac{\partial^{2}}{\partial \xi^{2}} \varphi_{L}=0
$$

where $\varphi_{\mathrm{R}}$ and $\varphi_{\mathrm{L}}$ are the first order amplitude of eqs. (47a) and $(47 b)$, respectively. These nonlinear evolution equations have been rederived by Mio et al. [33]. They have carried out analysis of the modulation instability of the Alfvén wave on the basis of eqs. (51 $a$ ) and (51b).

It would be worthwhile, however, to present here exact steady state solution of eqs. (51a) and (51b) obtained by Wadati et al. [34]. Substitution of a form

$$
\varphi_{\mathrm{R}}(\xi, \tau)=\sqrt{8} \psi(\xi, \tau) \exp \{i \chi(\xi, \tau)\}
$$

with real functions $\psi$ and $\chi$ into eq. (51a) yields a pair of coupled equations for $\psi$ and $\chi$ :

$$
\begin{aligned}
& \psi_{\tau}+6 \psi^{2} \psi_{\xi}+2 \mu \psi_{\xi} \chi_{\xi}+\mu \chi_{\xi \xi} \psi=0, \\
& \chi_{\tau} \psi+2 \chi_{\zeta} \psi^{3}-\mu \psi_{\xi \xi}+\mu \chi_{\xi}{ }_{\xi} \psi=0 .
\end{aligned}
$$

We seek a solution in the following form,

$$
\begin{gathered}
\chi(\xi, \tau)=\mu^{-1}(K \xi-\Omega \tau)+\theta(y), \\
\psi(\xi, \tau)=\psi(y),
\end{gathered}
$$

with

$$
y=\mu^{-1}(\xi-\lambda \tau),
$$

where wave number $K$, frequency $\Omega$ and propagation velocity $\lambda$ are constants to be determined from solutions of $(53 a)$ and $(53 b)$. Under the conditions of eqs. $(54 a) \sim(54 c)$, we can obtain

$$
\begin{aligned}
\left(\frac{\mathrm{d}}{\mathrm{d} y} \Phi\right)^{2}= & -\Phi^{4}+2 \lambda \Phi^{3}- \\
& -4\left\{\Omega+A+\frac{1}{4}(\lambda-2 K)^{2}-K^{2}\right\} \Phi^{2} \\
& +4 B \Phi-4 A^{2} \\
\frac{\mathrm{d}}{\mathrm{d} y} \theta= & \frac{A}{\Phi}+\frac{1}{2}(\lambda-2 K)-\frac{3}{2} \Phi
\end{aligned}
$$

where

$$
\Phi(y)=\psi^{2}(y)
$$

and, $A$ and $B$ are integration constants. Restricting our interest to solitary wave solutions which satisfy the boundary conditions

$$
\begin{aligned}
& \Phi(y) \rightarrow \Phi_{0}=\psi_{0}^{2}, \\
& \frac{\mathrm{d}}{\mathrm{d} y} \theta \rightarrow 0, \text { as }|y| \rightarrow \infty,
\end{aligned}
$$

we can specify the integral constants and the shift of carrier frequency as

$$
\begin{aligned}
& A=\frac{3}{2} \Phi_{0}^{2}-\frac{1}{2}(\lambda-2 K) \Phi_{0}, \\
& B=4 \Phi_{0}^{3}+\frac{1}{2}(12 K-5 \lambda) \Phi_{0}^{2}+2\left(K-\frac{1}{2} \lambda\right)^{2} \Phi_{0},
\end{aligned}
$$

$\Omega=K^{2}+2 K \Phi_{0}$

Straightforward but lengthy calculation gives

$$
\begin{aligned}
\Phi(y)=\psi^{2}(y) & =\Phi_{0}+\frac{8 \kappa \gamma^{2}}{\beta} \times \\
\times & {\left[\kappa m+\cosh \left(2 \gamma\left(y-y_{0}\right)\right)\right]^{-1 / 2}, }
\end{aligned}
$$




$$
\begin{aligned}
\theta(y)= & \theta\left(y_{0}\right)-3 \kappa \tan ^{-1} \times \\
& \times\left\{\sqrt{\frac{1-\kappa m}{1+\kappa m}} \tanh \left(\gamma\left(y-y_{0}\right)\right)\right\} \\
& -\gamma \delta \tan ^{-1}\left\{\sqrt{\frac{1-\kappa l}{1+\kappa l}} \tanh \left(\gamma\left(y-y_{0}\right)\right)\right\},
\end{aligned}
$$

where

$$
\begin{aligned}
& \kappa= \pm 1, \\
& l=\frac{\alpha}{\beta}+8 \frac{\gamma^{2}}{\beta \Phi_{0}} \text { and } m=\frac{\alpha}{\beta}, \\
& \alpha=2\left(2 \Phi_{0}-\lambda\right), \\
& \beta=4\left\{\left(\Phi_{0}+K\right)\left(\lambda-K-2 \Phi_{0}\right)\right\}^{1 / 2}, \\
& \gamma=\frac{1}{2}\left\{\left(\lambda-\lambda_{1}\right)\left(\lambda_{2}-\lambda\right)\right\}^{1 / 2}, \\
& \delta=\operatorname{sign} \text { of }\left(3 \Phi_{0}-\lambda+2 K\right) .
\end{aligned}
$$

The propagation velocity $\lambda$ is allowed to take a value in the region of

$$
\lambda_{1}<\lambda<\lambda_{2}
$$

where

$$
\begin{aligned}
& \lambda_{1}=2\left(K+2 \Phi_{0}\right)-2 \sqrt{\Phi_{0}\left(\Phi_{0}+K\right)}, \\
& \lambda_{2}=2\left(K+2 \Phi_{0}\right)+2 \sqrt{\Phi_{0}\left(\Phi_{0}+K\right)} .
\end{aligned}
$$

A similar analysis is possible for the left polarized waves. In the case, solitary waves are obtained just by replacing

$$
\Omega \rightarrow-\Omega \text { and } K \rightarrow-K
$$

in the above expressions, eqs. $(59 a) \sim(59 f)$ and eqs. $(60 b)$ and $(60 c)$. Then, we have an extra restriction on the wave number,

$$
\Phi_{0}>K
$$

As can be seen from eq. ( $58 a), \kappa=+1$ designates bright modulation while $\kappa=-1$ dark modulation of the amplitude, respectively. Eqs. (58a) and (58b) represent that modulation of the amplitude is closely coupled to modulation of the phase. Furthermore, unlike the envelope soliton given by eq. (37), the propagation velocity $\lambda$ of the solitary wave (58a) and $(58 b)$ is not an arbitrary constant, but it is restricted to a region defined by eqs. $(60 a) \sim(60 c)$. These properties are quite unique and could be detected by experiment. In figure 8 referring to the right polarized mode, we illustrate the bright Alfvén solitary wave and the dark Alfvén solitary wave for arbitrary choosen parameters of $\mu, K$ and $\Phi_{\mathrm{R}}$.

Besides the solitary waves discussed above, eqs. ( $51 a$ ) and $(51 b)$ admit also algebraic solitary waves. For the right polarized Alfvén wave, we get

$$
\begin{aligned}
\Phi_{\mathrm{R}}^{(a)}(y) & =\Phi_{0}+\frac{4 \gamma}{4+\gamma^{2}\left(y-y_{0}\right)^{2}} \\
\theta_{\mathrm{R}}^{(a)}(y) & =0\left(y_{0}\right)+\delta \tan ^{-1}\left(\varepsilon\left(y-y_{0}\right)\right)- \\
& -3 \tan ^{-1}\left(\frac{\gamma}{2}\left(y-y_{0}\right)\right)
\end{aligned}
$$

$$
\mu=0.5
$$

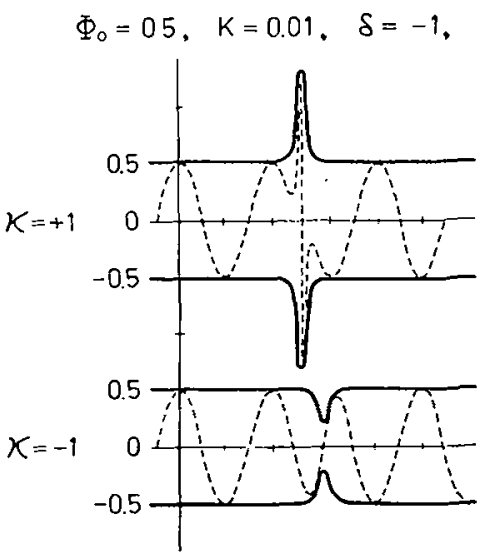

FIG. 8. - Envelope solitons of the right polarized Alfvén wave for arbitrary values of parameters $\mu=0.5$, with $\Phi_{0}=0.5$ and $K=0.01$. The upper trace is for the bright $(\kappa=+1)$ and the lower trace for the dark $(\kappa=-1)$ enveloppe solitons, respectively. The dotted line represents the real part of $\psi(\xi, \tau)$ $\exp (i \chi(\xi, \tau)$.

where

$$
\begin{aligned}
& \gamma=4\left(\Phi_{0}+K\right)+4 \delta \sqrt{\Phi_{0}\left(\Phi_{0}+K\right)}, \\
& \varepsilon=\frac{\gamma}{2}\left[2 \sqrt{\Phi_{0}+K}+\delta \sqrt{\Phi_{0}}\right]^{-1} \sqrt{\Phi_{0}}, \\
& \delta=+1 \text { for } \lambda_{+} \\
& -1 \text { for } \lambda_{-},
\end{aligned}
$$

and the velocities $\lambda \pm$ are defined as

$$
\lambda_{ \pm}=2\left(2 \Phi_{0}+K\right) \pm 2 \sqrt{\Phi_{0}\left(\Phi_{0}+K\right)} .
$$

Figure 9 illustrates the algebraic solitary Alfven wave with the right polarization at the velocity $\lambda_{+}$ and $\lambda_{-}$, respectively. For the left polarized Alfvén wave, we get

$$
\begin{aligned}
& \Phi_{\mathrm{L}}^{(a)}(y)=\Phi_{0}+\frac{4 \gamma}{4+\gamma^{2}\left(y-y_{0}\right)^{2}} \\
& \begin{aligned}
\theta_{\mathrm{L}}^{(a)}(y) & =0\left(y_{0}\right)-\tan ^{-1}\left(\varepsilon\left(y-y_{0}\right)\right)+ \\
& \quad+3 \tan ^{-1}\left(\frac{\gamma}{2}\left(y-y_{0}\right)\right)
\end{aligned}
\end{aligned}
$$

where

$$
\begin{aligned}
& \gamma=4\left(\Phi_{0}-K\right)+4 \sqrt{\Phi_{0}\left(\Phi_{0}-K\right)} \\
& \varepsilon=\frac{\gamma}{2}\left[2 \sqrt{\Phi_{0}-K}+\sqrt{\Phi_{0}}\right]^{-1} \sqrt{\Phi_{0}}
\end{aligned}
$$




$$
\mu=0.5
$$

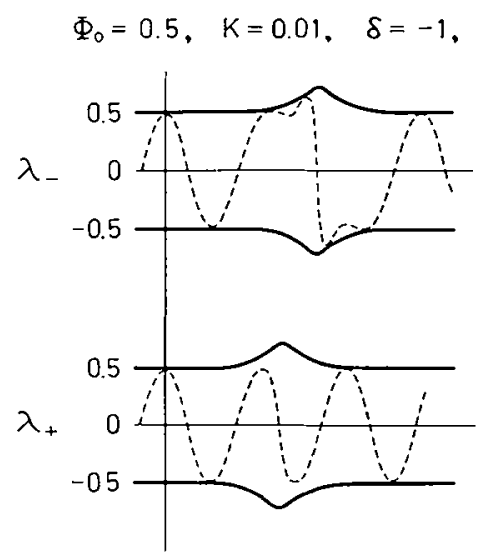

Fig. 9. - Algebraic envelope solitons of the right Alfvén wave.

with the velocity

$$
\lambda_{\mathrm{L}}=2\left(2 \Phi_{0}-K\right)+2 \sqrt{\Phi_{0}\left(\Phi_{0}-K\right)} .
$$

An algebraic envelope of the left polarized solitary Alfvén wave is shown in figure 10 . The figure indicates that contribution of a term with $\sin (\chi(\xi, \tau))$ is relatively large for this mode.

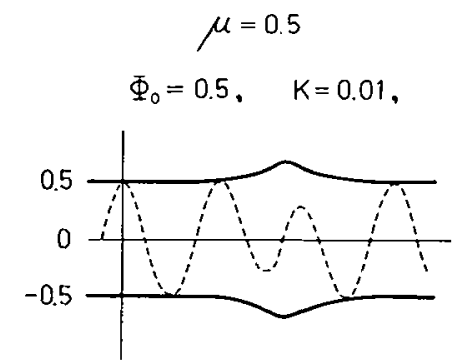

FIG. 10. - Algebraic envelope soliton of the left Alfvén wave.

We now close this section by emphasizing the above described peculiar properties of the solitary Alfvén waves are not known for any other types of nonlinear evolution equations.

6. Concluding discussions. - In the preceeding sections, we have discussed very fundamental aspects of solitons and envelope solitons in collisionless plasmas, restricting ourselves to their simplest forms. Our main purpose is firstly to emphasize that the theoretical studies of structure of the dressed solitons provide refined physical pictures on the nonlinear wave phenomena. Effects of the nonlinear wave interaction are classified to :

1) the self-interaction effects, of which the lowest order terms are essential to realize the solitons or the envelope solitons, while the higher order terms should be renormalized so as to remove the secular behaviour, and
2) the nonlinear mode-mode interaction effects, which are responsible to characterize dynamical processes of the nonlinear wave mode, such as the solitons or the envelope solitons.

Observed characteristics of the nonlinear wave phenomena will be subject to systematic analyses on the basis of conserved properties of the renormalized soliton cores and dynamical distortion of the coulds surrounding the cores.

Secondly, reffering to the ion plasma wave, we have discussed nonlinear wave modulation of the strongly dispersive waves with account of the effects of resonant particles at the group velocity. These particles are expected to play important role in heating processes of plasmas by large amplitude waves. Higher order effects on the envelope solitons have been discussed by us [35], and recently Kodama [36] has presented a renormalization procedure for a model system describing a strongly dispersive wave.

Thirdly, we have presented some detailed discussions on a new type of nonlinear evolution, which has been derived for the circular polarized Alfvén waves. Rigorous steady state solutions present quite exotic envelope solitons. Since this equation has not been examined so far on the frame-work of the inverse scattering method, we call attentions of theoretical researchers working in this field $\left({ }^{2}\right)$.

We conclude present paper that now the self-interaction effects of coherent nonlinear waves have been well understood owing to advancement of theoretical studies. These coherent nonlinear waves will be also playing crucial role in the anomalous transport processes encountered in various high temperature plasma devices, where the processes have been phenomenologically treated on a basis of the concept of quasi-linear theory. Although we admit practical convenience of these approaches for supplying a conceptual guidance, theoretical endeavor for deeper understanding of the fundamental properties of nonlinear wave phenomena is indispensable to establish the solid grounds for researches of such complicated nonlinear wave-particles system as collisionless plasmas.

Acknowledgments. - We are heartly obliged to Professor K. Takayama for his constant encouragement extended for us. We wish to express their sincere thanks to Professor T. Taniuti, Professor M. Toda and Professor N. Yajima for their valuable discussions given to us.

(2) At the conference, D. J. Kaup has commented that he and Newell have solved this type of nonlinear evolution equation on the basis of a new scheme of the inverse scattering transformation method. We remark, however, that their boundary conditions (amplitude vanishes at the infinity) differs from the present ones, eqs. (56a) and (56b). 


\section{Réferrences}

[1] Scotr-Russel, J., Proc. Roy. Soc. Edinburgh (1844) 319.

[2] Korteweg, D. J. and de Vries, G., Phil. Mag. 39 (1895) 422.

[3] Gardner, C. S. and Morikawa, G. M., Courant Inst. of Math. Sdi. Rept. NYO-9082, 1969 (unpublished); Comm. Pure Appl. Math. 18 (1965) 35.

[4] Washimi, H. and TANiuti, T., Phys. Rev. Lett. 17 (1966) 996.

[5] Ikezi, H., Taylor, R. J. and Baker, R. D., Phys. Rev. Lett. 25 (1970) 11.

[6] Ikezi, H., Phys. Fluids 16 (1973) 1668.

[7] Gardner, C. S., Green, J. M., Kruskal, M. D. and Miura, R. M., Phys. Rev. Lett. 19 (1967) 1095.

[8] Arlowitz, M. J., Kaup, D. J., Newell, A. C. and Segur, H., Studies Appl. Math. L111 (1974) 249.

[9] Taniuti, T., Prog. Theor. Phys. Suppl. (1974) No. 55.1

[10] Kato, Y., TaJiri, M. and TaniUti, Phys. Fluids 15 (1972) 865.

[11] Watanabe, S., J. Plasma Phys. 14 (1975) 353.

[12] Watanabe, S., J. Plasma Phys. 15 (1975) 67.

[13] TAppert, F. D., Phys. Fluids 15 (1972) 2446.

[14] Tagare, S. G., Plasma Phys. 15 (1973) 1247.

[15] SChamel, H., J. Plasma Phys. 9 (1973) 377.

[16] Konno, K. and Ichikawa, Y. H., J. Phys. Soc. Japan 37 (1974) 1631.

[17] IChikawa, Y. H., Mitsuhashi, T. and Konno, K., J. Phys. Soc. Japan 41 (1976) 1382.

[18] Konno, K., Mitsuhashi, T. and Ichikawa, Y. H., J. Phys. Soc. Japan 43 (1977) 669.

[19] Sugimoto, N. and KaKutani, T., report at Theoretical Meeting on Dynamical Processes of Dressed Solitons, held at Institute of Plasma Physics, Nagoya (June lst $\sim$ 2nd, 1977).

[20] Kodama, Y. and TANiUTi, T., report at the same meeting reffered in [19] ; preprint DPNU-20, 1977.
[21] Taniuti, T. and Yajima, N., J. Math. Phys. 10 (1969) 1369.

[22] Shimizu, K. and IChikawa, Y. H., J. Phys. Soc. Japan, 33 (1973) 789.

[23] Ikezi, H. and Kiwamoto, Y., Phys. Rev. Lett. 27 (1971) 718.

[24] Ichikawa, Y. H. and Taniuti, T., J. Phys. Soc. Japan 34 (1973) 513.

[25] Watanabe, S., J. Plasma Phys. 17 (1977) 487.

[26] Ikezi, H., Schwarzenegger, K., Kamimura, T. and OHSAWA, N., private communication.

[27] Yajima, N., Oikawa, M., Satsuma, J. and Namba, C., Reports of Res. Inst. for Applied Mechanics (Kyushu University), 1975, 22, 70.

[28] Hasegawa, A. and Chen, L., Phys. Rev. Lett. 32 (1974) 454.

[29] Belcher, J. W. and DAvis, L., J. Geophys. Res. 76 (1971) 3534.

[30] Hasegawa, A. and Mima, K., Phys. Rev. Lett. 37 (1976) 690.

[31] Kakutani, T. and Ono, H., J. Phys. Soc. Japan 26 (1969) 1305.

[32] Kawahara, T., Seminar Notes from R. I. M. S. of Kyoto University, 1973, No. 187.

[33] Mio, K., Ogino, T., Minami, K. and TAKeda, S., $J$. Phys. Soc. 41 (1976) 265.

[34] Wadati, M., Sanuki, H., Konno, K. and Ichikawa, Y. H., the Proc. of Conference on Solitons, Rocky Mountain Math. Journal, 1977, to be published.

[35] Ichikawa, Y. H., Mitsuhashi, T. and Konno, K., $J$. Phys. Soc. Japan 43 (1977) 675.

[36] Kodama, Y., preprint DPNU-22, 1977. 